\title{
Spectrum of Corrugated and Periodically Loaded Waveguides from Classical Matrix Eigenvalues
}

\author{
Smain Amari, Member, IEEE, Rüdiger Vahldieck, Fellow, IEEE, Jens Bornemann, Senior Member, IEEE, and \\ Pascal Leuchtmann
}

\begin{abstract}
The paper presents a rigorous full-wave analysis of propagation in corrugated and periodically loaded waveguides. The propagation constants are determined from the classical eigenvalues of a canonical matrix eigenvalue problem instead of a determinant. The entries of the matrix are computed only once per frequency point. The entire $k_{0}-\beta$ diagram of a corrugated circular waveguide, a circular waveguide periodically loaded with dielectric disks, and a rectangular waveguide periodically loaded with capacitive irises are determined and compared with results of other researchers. Excellent agreement is documented in each case.
\end{abstract}

Index Terms-Eigenvalues, integral equations, modal analysis, periodic structures.

\section{INTRODUCTION}

$\mathbf{P}$ ERIODIC structures have been investigated by many researchers due to their importance in slow-wave structures, backward-wave oscillators, corrugated antennas and antenna feeds, phase shifters, polarizers, and even transmission systems [1]-[18]. The recent discovery of photonic-bandgap materials has created a demand for accurate and efficient methods of analysis and design of periodic structures [19]-[23]. In addition, modern satellite communications often require corrugated antennas and feeds with high symmetry and low cross polarization in the radiation pattern [13], [16]. Accurate prediction of the propagation behavior of periodic structures is an essential step in the successful design of these systems. A variety of numerical techniques have been used to achieve this goal. In broad terms, these belong to one of two groups: 1) methods which determine the propagation constants of the Floquet modes from a nonlinear (determinant) equation, and 2) methods which determine the propagation constants of the Floquet modes from the classical eigenvalues of a matrix.

Among the methods in the first group are those based on expansions in space-harmonics and the surface impedance approach [13]. In these methods, the propagation constants are determined as the roots of a nonlinear determinant equation following an iterative process. Such an approach can be time consuming and suffers the risk of missing degenerate or nearly degenerate roots. In addition, complex modes introduce an additional complexity as they require two-dimensional search algorithms to locate the real and imaginary parts of the propaga-

Manuscript received September 21, 1999; revised December 13, 1999.

S. Amari and J. Bornemann are with the Department of Electrical and Computer Engineering, University of Victoria, Victoria, B.C. Canada V8W 3P6.

R. Vahldieck and P. Leuchtmann are with the Swiss Federal Institute of Technology, ETH, Zürich, Switzerland.

Publisher Item Identifier S 0018-9480(00)02049-4. tion constant. A more efficient method of this group was presented by Esteban and Rebollar [15]. In their approach, which takes into account the finite thickness of the corrugations, the impedance-matrix formulation of the mode-matching technique (MMT) was used to derive a determinant equation from a matrix of reduced size [15]. Furthermore, the determinant is shown to be a polynomial in the propagation constant; this feature is used to further improve the search for the roots [15].

The advantages of methods in the second group are well described by Davies in connection with the finite-element method (FEM) [18, pp. 8-11]. Davies argues that methods that determine the propagation constants from the classical eigenvalues of a matrix are possibly the most efficient methods provided other criteria regarding absence of spurious roots and sparsity of matrices are met [18]. In addition to the FEM, propagation in waveguides periodically loaded with irises of zero-thickness has been analyzed by Collin [3] using modal expansions leading to a classical matrix eigenvalue problem. Similar formulation of the resonant zero-thickness iris in a rectangular waveguide was also presented in [10].

In this paper, we present a formulation that allows the complete spectrum of periodically loaded waveguides to be determined from the classical eigenvalues of a canonical matrix eigenvalue problem. Propagating, evanescent, and complex modes, should the latter be present, are determined accurately and efficiently. The analytic content of the approach makes it more efficient, but not as general as the FEM [18]. The approach is based on the coupled-integral-equation technique (CIET), which has the analytical content of the MMT, in addition to providing a simple mechanism to include a priori information such as the edge condition [24], [25].

The approach, which has already been applied to TE modes with no angular dependence in corrugated circular waveguides [26], is here applied to the propagation in a corrugated circular waveguide, a circular waveguide periodically loaded with dielectric disks, as well as a rectangular waveguide periodically loaded with capacitive irises. Numerical results are compared with those of other researchers to demonstrate its accuracy and efficiency.

\section{THEORY}

Consider a periodically loaded waveguide, as shown in Fig. 1(a). The unit cell of length $p$ comprises two different regions I and II of width $t$ and $g$, respectively. We assume that the normal modes of the two regions have already been determined using any of the numerous numerical methods [27]. For the corrugated waveguide [see Fig. 1(b) and (d)], these are 


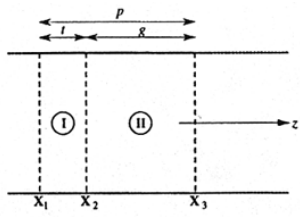

a)

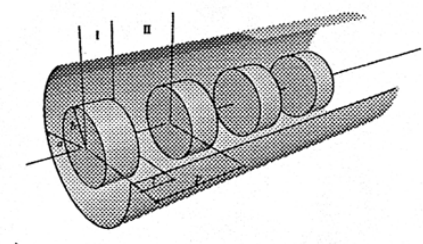

c)

d)

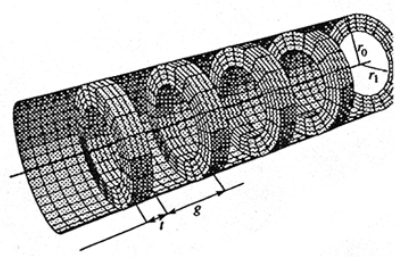

b)

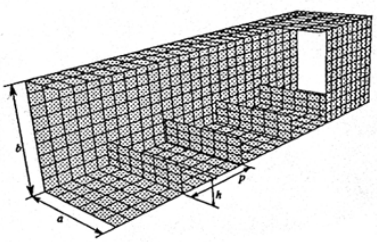

Fig. 1. (a) Cross section and side view of a periodically loaded waveguide. The unit cell of width $p$ comprises two regions of thickness $t$ and $g$. (b) Corrugated circular waveguide. (c) Circular waveguide loaded with dielectric disks. (d) Rectangular waveguide loaded with capacitive irises.

known analytically, for the dielectric loaded waveguide, these are determined straightforwardly [28]. For simplicity, we focus attention on the corrugated waveguide shown in Figs. 1(b) and (d) can be handled with minor modifications to deal with the nonhomogeneity of the dielectric loaded region.

Following the CIET, the transverse electric and magnetic fields in regions I and II are expanded in series of the normal modes of the two regions [24]. Let $\mathbf{e}_{n}^{i}$ denote the transverse electric field of the $n$th normal mode of the $i$ th region, $k_{n z}^{i}$ its propagation constant, and $Y_{n}^{i}$ its wave admittance. The transverse magnetic field of this mode is written as $Y_{n}^{i}\left(\mathbf{e}_{z} \times \mathbf{e}_{n}^{i}\right)$ where $\mathbf{e}_{z}$ is the unit vector in the $z$-direction (direction of propagation) [3, p. 174]. We also assume that the transverse functions $\mathbf{e}_{n}^{i}$ are normalized according to

$$
\iint_{S_{i}} \mathbf{e}_{n}^{i} \cdot \mathbf{e}_{m}^{i} d s_{i}=\delta_{n m}
$$

where $S_{i}$ is the cross section of region $i$. In the $i$ th region, the transverse electric and magnetic fields are expanded in series of the form

$$
\mathbf{E}_{t}^{i}=\sum_{n=1}^{\infty}\left(a_{n}^{i} e^{-j k_{n z}^{i} z}+b_{n}^{i} e^{j k_{n z}^{i} z}\right) \mathbf{e}_{n}^{i}
$$

and

$$
\mathbf{H}_{t}^{i}=-\sum_{n=1}^{\infty} Y_{n}^{i}\left(a_{n} e^{-j k_{n z} z}-b_{n}^{i} e^{j k_{n z}^{i} z}\right)\left(\mathbf{e}_{z} \times \mathbf{e}_{n}^{i}\right) .
$$

Here, $i=\mathrm{I}$, II. Let us furthermore assume that the true distribution of the transverse electric field at $z=0, z=t$, and $z=p=t+g$ are given by three unknown vector functions $\mathbf{X}_{1}$, $\mathbf{X}_{2}$, and $\mathbf{X}_{3}$, respectively.

In a periodic structure of period $p$, a generic component $F(z)$ of the electromagnetic field satisfies the Floquet condition [3, p. 370]

$$
F(z+p)=e^{-\gamma p} F(z) \quad \forall z
$$

Here, $\gamma$ is the propagation constant of the Floquet mode. The functions $\mathbf{X}_{1}$ and $\mathbf{X}_{3}$ are, therefore, related by

$$
\mathbf{X}_{3}=e^{-\gamma p} \mathbf{X}_{1}
$$

When the unit cell exhibits an additional symmetry, such as a glide-reflection symmetry, for example, the two functions $\mathbf{X}_{1}$ and $\mathbf{X}_{2}$ are also related through this symmetry. In such a case, only one unknown function remains to be determined; this was the subject of a recent paper on a periodically loaded parallelplate waveguide with gilde reflection symmetry [29].

When the functions $\mathbf{X}_{1}$ and $\mathbf{X}_{2}$ are not related by a symmetry operation, the discussion presented in [29] does not apply; an alternative transformation of the coupled integral equations is presented here to deal with this more general case.

To determine the remaining two functions $\mathbf{X}_{1}$ and $\mathbf{X}_{2}$, we first use the boundary conditions of the electric field to eliminate the expansion coefficients $a_{n}^{i}$ and $b_{n}^{i}$ in favor of $\mathbf{X}_{1}$ and $\mathbf{X}_{2}$ and then enforce the continuity of the transverse magnetic field at $z=t$ and its Floquet condition at $z=p$. Two coupled vector integral equations in $\mathbf{X}_{1}$ and $\mathbf{X}_{2}$ result. The details are not included here, but can be found in [24]; we only present the two equations that take the form

$$
\begin{aligned}
\sum_{n=1}^{\infty} Y_{n}^{\mathrm{II}} \frac{\tilde{X}_{2}^{\mathrm{II}}(n) e^{\gamma p}-\tilde{X}_{1}^{\mathrm{II}}(n) \cos \left(k_{n z}^{\mathrm{II}} g\right)}{\sin \left(k_{n z}^{\mathrm{II}} g\right)}\left(\mathbf{e}_{z} \times \mathbf{e}_{n}^{\mathrm{II}}\right) \\
\quad=\sum_{n=1}^{\infty} Y_{n}^{\mathrm{I}} \frac{\tilde{X}_{1}^{\mathrm{I}}(n) \cos \left(k_{n z}^{\mathrm{I}} t\right)-\tilde{X}_{2}^{\mathrm{I}}(n)}{\sin \left(k_{n z}^{\mathrm{I}} t\right)}\left(\mathbf{e}_{z} \times \mathbf{e}_{n}^{\mathrm{I}}\right)
\end{aligned}
$$

and

$$
\begin{aligned}
\sum_{n=1}^{\infty} Y_{n}^{\mathrm{II}} & \frac{\tilde{X}_{2}^{\mathrm{II}}(n) \cos \left(k_{n z}^{\mathrm{II}} g\right)-e^{-\gamma p} \tilde{X}_{1}^{\mathrm{II}}(n)}{\sin \left(k_{n z}^{\mathrm{II}} g\right)}\left(\mathbf{e}_{z} \times \mathbf{e}_{n}^{\mathrm{II}}\right) \\
& =\sum_{n=1}^{\infty} Y_{n}^{\mathrm{I}} \frac{\tilde{X}_{1}^{\mathrm{I}}(n)-\tilde{X}_{2}^{\mathrm{I}}(n) \cos \left(k_{n z}^{\mathrm{I}} t\right)}{\sin \left(k_{n z}^{\mathrm{I}} t\right)}\left(\mathbf{e}_{z} \times \mathbf{e}_{n}^{\mathrm{I}}\right) .
\end{aligned}
$$

The transformations $\tilde{X}_{j}^{\mathrm{I}}(n)$ and $\tilde{X}_{j}^{\mathrm{II}}(n)$ are given by

$$
\tilde{X}_{j}^{i}(n)=\iint_{S_{1}} \mathbf{X}_{j} \cdot \mathbf{e}_{n}^{i} d s_{1}, \quad j=1,2 ; \quad i=\mathrm{I}, \mathrm{II}
$$

where $S_{1}$ is the cross section of region I. To solve these integral equations, we expand the two functions in a series of appropriate basis functions

$$
\mathbf{X}_{1}=\sum_{p=1}^{M} c_{p} \mathbf{Q}_{p}
$$

and

$$
\mathbf{X}_{2}=\sum_{p=1}^{M} d_{p} \mathbf{Q}_{p} .
$$

The same basis functions were used since the two discontinuities have the same geometry. Substituting these expansions in the integral equations and applying the moment method [30], 
we get a set of linear equations in the coefficients $c_{p}$ and $d_{p}$, namely,

$$
\left[\begin{array}{ll}
A & B \\
B & A
\end{array}\right]\left[\begin{array}{l}
c \\
d
\end{array}\right]+\left[\begin{array}{lc}
G e^{-\gamma p} & 0 \\
0 & G e^{\gamma p}
\end{array}\right]\left[\begin{array}{l}
c \\
d
\end{array}\right]=0
$$

The entries of the matrices are given by

$$
\begin{aligned}
& A_{i j}=\sum_{n=1}^{\infty} Y_{n}^{\mathrm{I}} \frac{\tilde{Q}_{i}^{\mathrm{I}}(n) \tilde{Q}_{j}^{\mathrm{I}}(n)}{\sin \left(k_{z n}^{\mathrm{I}} t\right)} \\
& B_{i j}=-\sum_{n=1}^{\infty} Y_{n}^{\mathrm{I}} \frac{\tilde{Q}_{i}^{\mathrm{I}}(n) \tilde{Q}_{j}^{\mathrm{I}}(n)}{\tan \left(k_{z n}^{\mathrm{I}} t\right)}-\sum_{n=1}^{\infty} Y_{n}^{\mathrm{II}} \frac{\tilde{Q}_{i}^{\mathrm{II}}(n) \tilde{Q}_{j}^{\mathrm{II}}(n)}{\tan \left(k_{z n}^{\mathrm{II}} g\right)}
\end{aligned}
$$

and

$$
G_{i j}=\sum_{n=1}^{\infty} Y_{n}^{\mathrm{II}} \frac{\tilde{Q}_{i}^{\mathrm{II}}(n) \tilde{Q}_{j}^{\mathrm{II}}(n)}{\sin \left(k_{z n}^{\mathrm{II}} g\right)} .
$$

Note that matrices $[A],[B]$, and $[G]$ are symmetric and real since, for lossless structures, the wave admittance is either proportional or inversely proportional to $k_{z n}$ [3]; the ratio of the wave admittance and trigonometric functions remains real. These features are exploited in reducing the numerical effort and avoiding complex arithmetic.

Equation (11) is not in a convenient form as it contains two different functions of $\gamma$, namely $e^{\gamma p}$ and $e^{-\gamma p}$; a direct solution would lead to a nonlinear determinant equation, which we set out to avoid.

We first eliminate the vector $[d]$ from (11) to get a reduced equation in terms of $[c]$ only, or

$$
[R][c]+[S][c] e^{\gamma p}+[S]^{t}[c] e^{-\gamma p}=0 .
$$

The matrices in this equation are given by

$$
[R]=A B^{-1} A+G B^{-1} G-B
$$

and

$$
[S]=A B^{-1} G \quad[S]^{t}=G B^{-1} A .
$$

Here, $[S]^{t}$ is the transpose of $[S]$.

Let $\lambda=e^{\gamma p}$, and note that $e^{-\gamma p}=1 / \lambda$, then (15) takes the form

$$
\lambda^{2}[S][c]+\lambda[R][c]+[S]^{t}[c]=0 .
$$

Let us introduce a vector $[v]$ of the same dimension as $[c]$ such that

$$
[v]=\lambda[c] .
$$

The eigenvalue equation, i.e., (18), is finally rewritten in the more convenient form [31]

$$
\left[\begin{array}{cc}
R & S^{t} \\
-I & 0
\end{array}\right]\left[\begin{array}{l}
v \\
c
\end{array}\right]+\lambda\left[\begin{array}{ll}
S & 0 \\
0 & I
\end{array}\right]\left[\begin{array}{l}
v \\
c
\end{array}\right]=0 .
$$

Here, $I$ is the identity matrix of order $M \times M$. Equation (20) is in the classical canonical form $[A][x]+\lambda[B][x]=0$; its eigenvalues and, consequently, the propagation constants, can be straightforwardly and reliably determined using commercially available software packages.

Note that the entries of the matrices are calculated only once for each frequency. When regions I and II have homogeneous cross sections, such as Fig. 1(b), it is further advantageous to compute the transforms of the basis functions [see (8)] only once, as they do not depend on frequency, and store them in the computer for later use.

Once the eigenvalues $\lambda$ have been determined, the real and imaginary parts of the propagation constant $\gamma=\alpha+j \beta$ are given by

$$
\alpha p=\ln |\lambda| \quad \beta p=\angle \lambda .
$$

Since $\lambda$ is not changed under an arbitrary shift $\beta p \rightarrow \beta p+2 n \pi$, the dispersion diagram is periodic in $\beta p$ with period $2 \pi$, a well known result for periodic structures. It is, therefore, sufficient to determine the dispersion diagram in the interval $-\pi \leq \beta p \leq \pi$.

In the numerical solution, appropriate basis functions are needed. The simplest choice is to use the modes of region I as basis functions. For such a choice, the entries of the matrices take simple forms due to the orthogonality of the normal modes [3]. If the edge condition is found to be dominant, basis functions that include the edge conditions can be constructed by introducing a proper weighting factor [32] or by locally solving Maxwell's equations [33]. For example, suitable basis functions which include the edge condition at the $90^{\circ}$ metallic wedge of Fig. 1(b) are given by

$$
\mathbf{Q}_{p}=\frac{\mathbf{e}_{p}^{\mathrm{I}}}{\left[1-\left(\frac{\rho}{r_{1}}\right)^{2}\right]^{1 / 3}} .
$$

\section{RESULTS}

The approach presented has been applied to a variety of periodically loaded waveguides, and here we report the results for a few of them.

\section{A. Corrugated Circular Waveguide}

We focus attention on hybrid modes with unity angular dependence, as these are the relevant modes for corrugated antennas and antenna feeds [13]. The normal modes of Sections I and II are expressible in terms of Bessel functions and can be found, for example, in [28]. Both basis functions with and without the edge conditions were used and converged to the same result, although a smaller number of basis functions with the edge conditions are required, especially for strongly corrugated waveguides. All numerical results presented in this section were obtained using three basis functions and summing $32 \mathrm{TE}$ and $32 \mathrm{TM}$ modes in computing the entries of the matrices in (12)-(14).

To validate the theory and computer code, we determined the real and imaginary parts of the propagation constant of a structure analyzed by Esteban and Rebollar [15]. Fig. 2 shows the imaginary part of the propagation constant as a function of frequency for a corrugated waveguide when $t=0.01 \mathrm{~mm}$, $g=1 \mathrm{~mm}, r_{1}=10 \mathrm{~mm}$, and $r_{1} / r_{0}=0.9$. The circles are from 


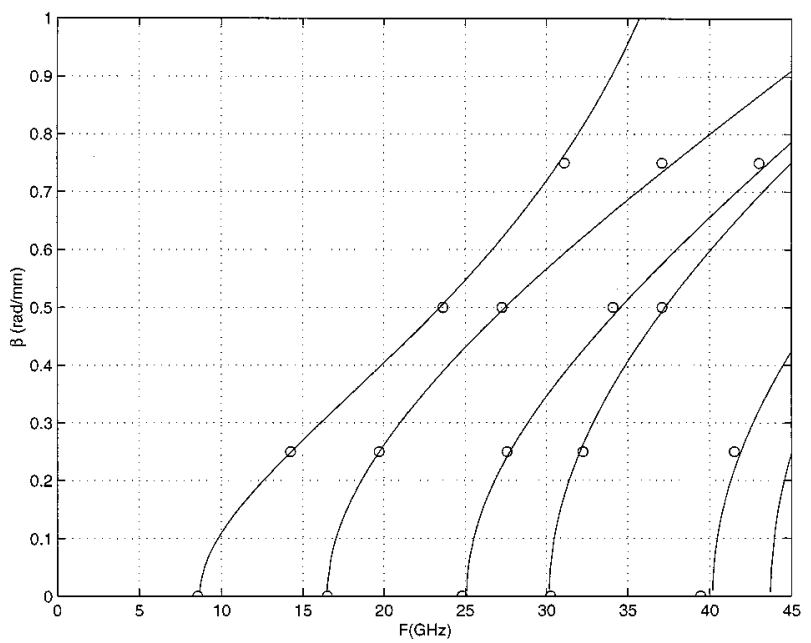

Fig. 2. Dispersion behavior of a circular corrugated waveguide. $r_{1}=10 \mathrm{~mm}$, $t=0.01 \mathrm{~mm}, g=1$. $\mathrm{mm}$, and $r_{1} / r_{0}=0.9$. The circles are from [15].

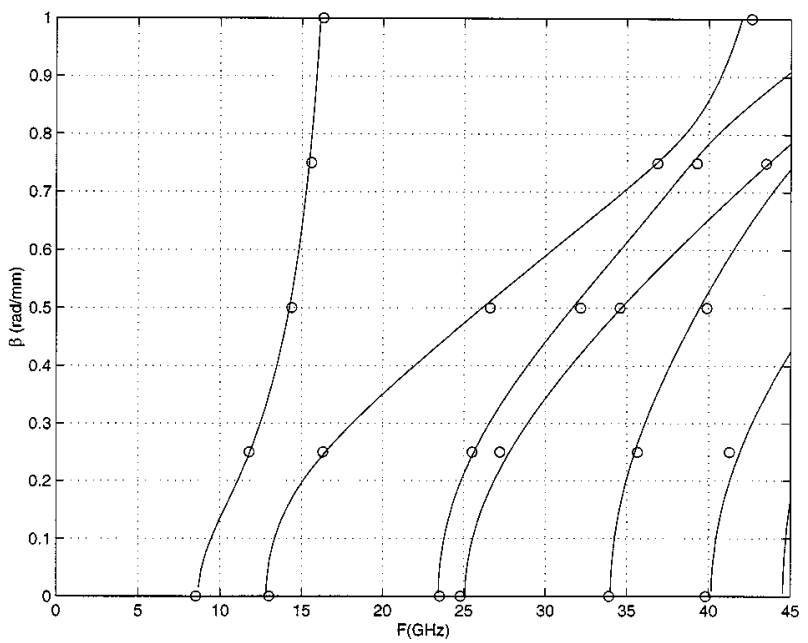

Fig. 3. Dispersion behavior of a circular corrugated waveguide. $r_{1}=10 \mathrm{~mm}$, $t=0.01 \mathrm{~mm}, g=1 . \mathrm{mm}$, and $r_{1} / r_{0}=0.7$. The circles are from [15].

[15]. Except for the mode starting at $44 \mathrm{GHz}$, and which was not reported in [15], the agreement between the two results is excellent.

Fig. 3 shows the same diagram when $r_{1} / r_{0}=0.7$. The circles are again from [15]. Except for the higher mode, the agreement between the two calculations is excellent.

A further test of the approach was carried out by computing both the real and imaginary parts of the propagation constant for propagating, evanescent and complex modes. In fact, all these modes are computed simultaneously from the eigenvalues of the canonical matrix [see (20)]. Fig. 4 shows a plot of $\beta$ and $\alpha$ versus normalized frequency when $t=0.01 \mathrm{~mm}, g=1 \mathrm{~mm}, r_{1}=$ $10 \mathrm{~mm}$, and $r_{1} / r_{0}=0.4$. The dashed lines represent complex modes where both $\alpha$ and $\beta$ are nonzero. The stars are from [15]. The agreement between the two results is good, including the presence of complex modes.

The speed of the present method allows us to determine the entire dispersion diagram of the structure with minimum numerical effort. Fig. 5 shows a $k_{0}-\beta$ diagram when $t=4 \mathrm{~mm}$, $g=5 \mathrm{~mm}, r_{1}=10 \mathrm{~mm}$, and $r_{1} / r_{0}=0.4$. The presence of

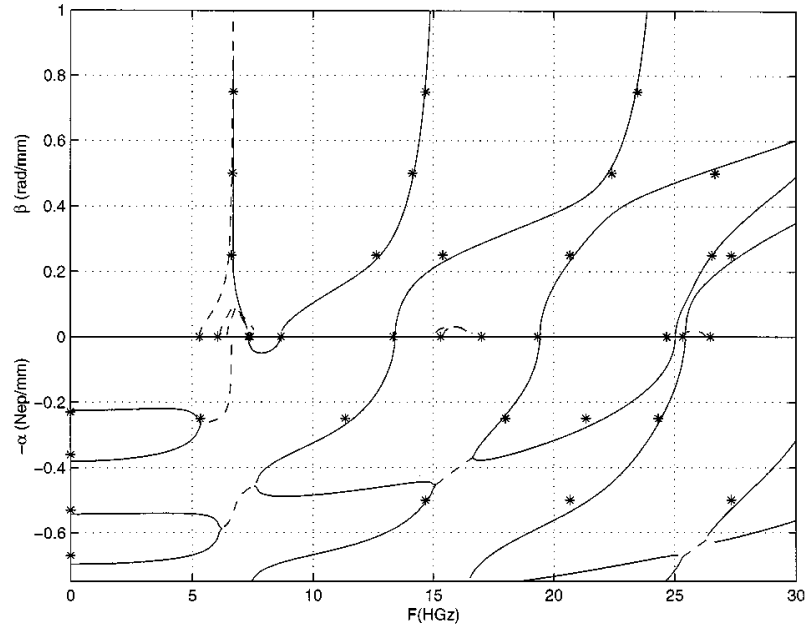

Fig. 4. Real $\alpha$ and imaginary $\beta$ parts of $\gamma$ versus normalized frequency for a circular corrugated waveguide when $r_{1} / r_{0}=0.4, r_{1}=10 \mathrm{~mm}, t=0.01$ $\mathrm{mm}$, and $g=1 \mathrm{~mm}$. The stars are from [15].

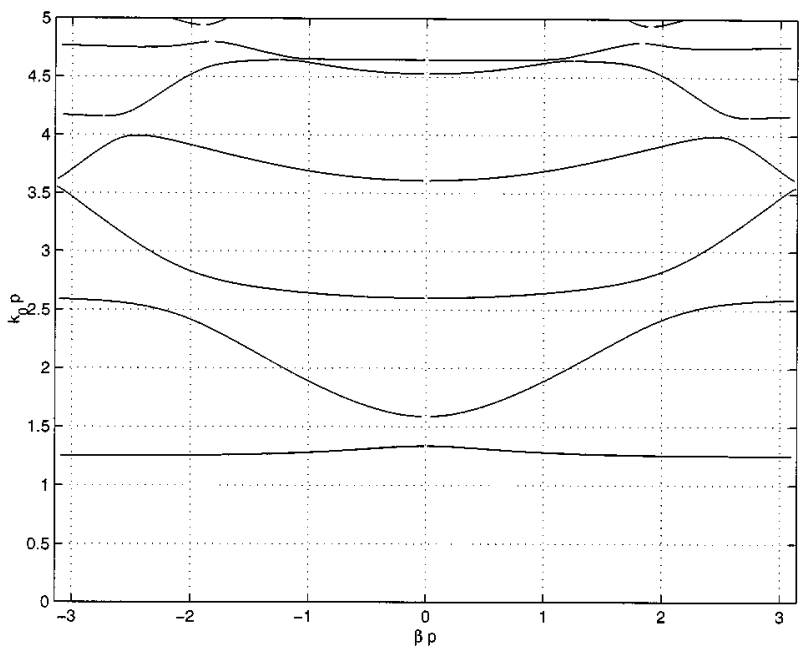

Fig. 5. $k_{0}-\beta$ diagram of a corrugated circular when $r_{1} / r_{0}=0.4, r_{1}=10$ $\mathrm{mm}, t=4 \mathrm{~mm}$, and $g=5 \mathrm{~mm}$. Note the presence of the bandgaps in the diagram.

the bandgaps, separated by passbands, is clearly visible. Note also that the group velocity of the lowest mode is negative at $\beta=0$. It turns out that the group velocity of the lowest mode is very sensitive to the depth of the corrugations, a point extensively illustrated by Clarricoats and coworkers and confirmed by our calculations [13]. Fig. 6 shows the first branch in the dispersion diagram (with unity angular dependence) for four different values of the ratio $r_{1} / r_{0}$. The change in sign of the group velocity at $\beta=0$ as the depth of the corrugation increases is well demonstrated. The first branch supports backward waves for strongly corrugated waveguides.

\section{B. Waveguide Loaded with Dielectric Rings}

The second structure investigated consists of a circular waveguide periodically loaded with dielectric disks [see Fig. 1(c)]. In this case, the modes of the dielectric loaded region are determined numerically following the presentation of Harrington [28]. We also limit the analysis to TE modes with no angular 


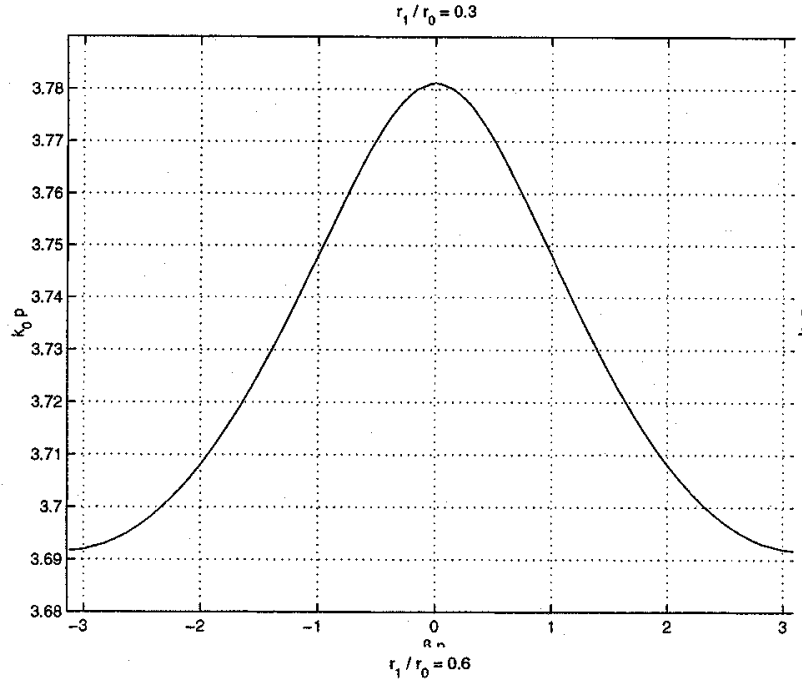

(a)

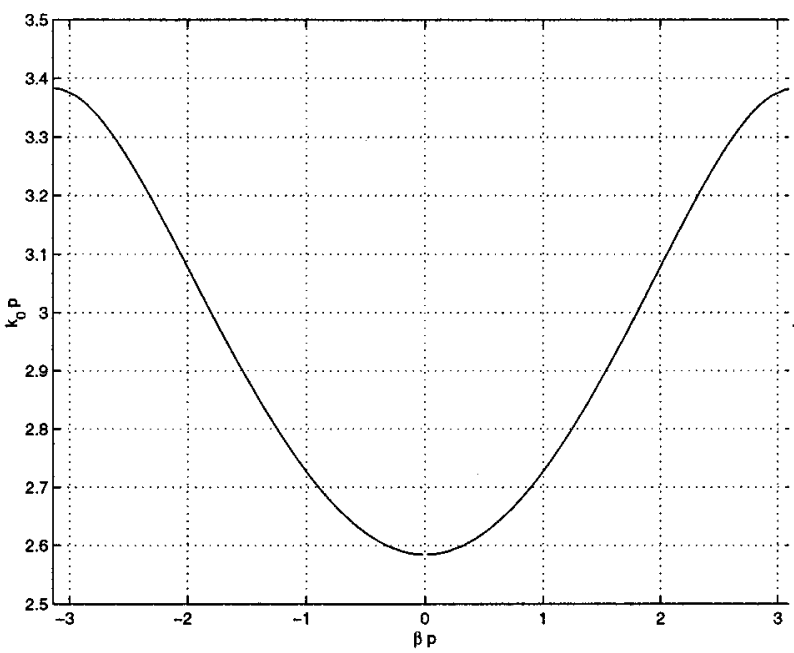

(c)

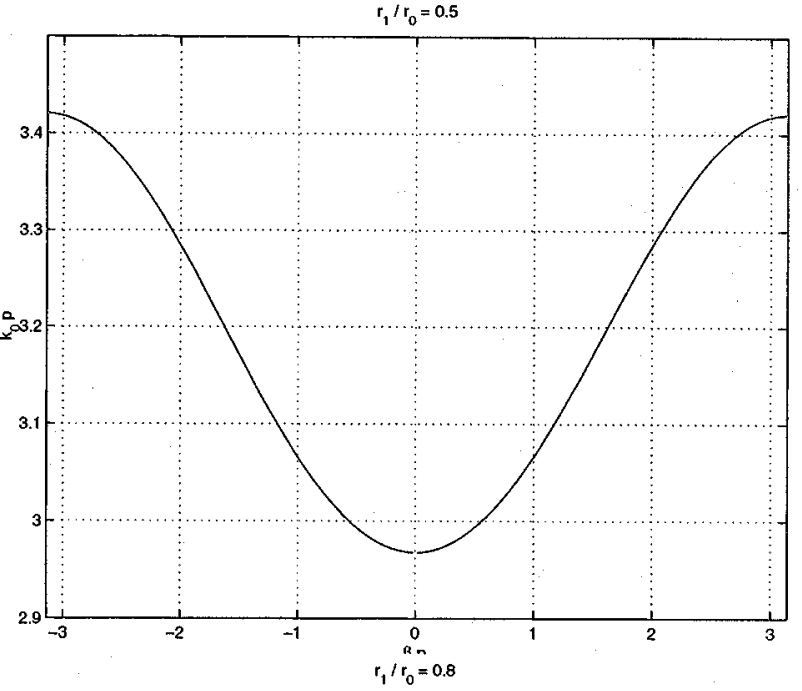

(b)

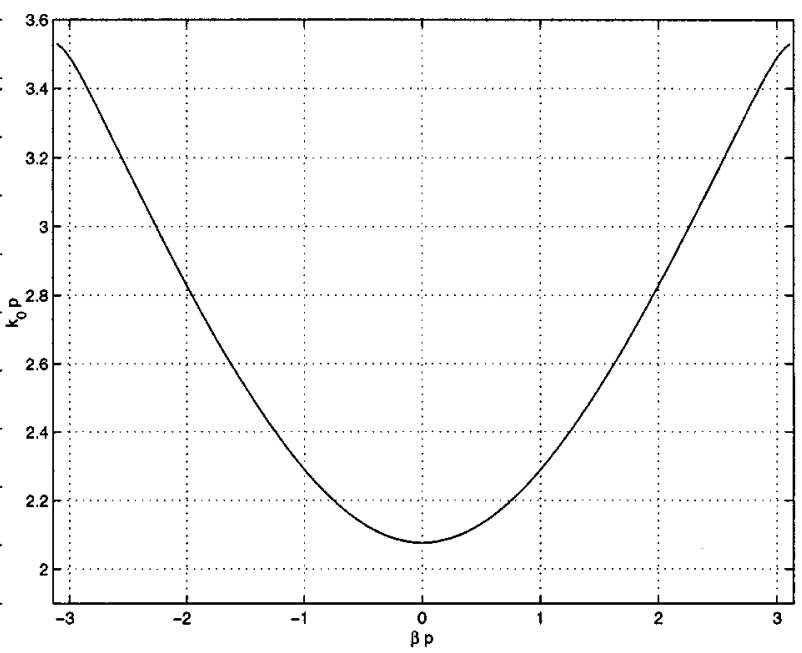

(d)

Fig. 6. First branch in $k_{0}-\beta$ diagram of a circular waveguide for four values of the ratio $r_{1} / r_{0} \cdot p=r_{0}, t=r_{0} / 5$. Note the change in sign in the group velocity of deep corrugations $\left(r_{1} / r_{0}=0.3\right)$.

dependence given the importance of these modes in $\mathrm{TE}_{01 \delta} \mathrm{di}-$ electric resonator filters.

To establish the validity of the approach, we determine the dispersion diagram of a structure where the dielectric constant of the disks approaches unity and compare with the analytical results of an empty circular waveguide. Fig. 7 shows both numerical and analytical results with $\epsilon_{r}=1$. The agreement between the two is excellent. These results were obtained from three basis functions and 30 terms were summed in computing the entries of the matrices. Note that both diagrams are plotted using the reduced Brillouin zone [1].

In the limit $t \rightarrow p$, the dispersion diagram of the periodic structure should approach that of a circular waveguide loaded with a dielectric rod of dielectric constant $\epsilon_{r}$. The numerical results obtained when $t=0.99 p$ indeed satisfy this observation [8]. A more practical situation corresponding to a disk with dielectric constant $\epsilon_{r}=10$ was also analyzed; its $k_{0}-\beta$ diagram is shown in Fig. 8. The presence of stopbands separated by passbands is clearly visible. There is no propagation in the range of frequencies $2.144 \leq k_{0} p \leq 3.124$ and $3.436 \leq k_{0} p \leq 3.767$ although other modes with different angular dependence might be propagating in this frequency range. The effect of the dielectric loading on the dispersion diagram is also visible when compared with the empty waveguide of Fig. 7. The addition of the dielectric disks lowers the cutoff frequency and flattens the branches of the dispersion diagram resulting in slowly changing group velocities. Although the first branch exhibits a positive group velocity, other higher branches support waves with negative group velocities, which are essential to devices such as backward-wave oscillators. 


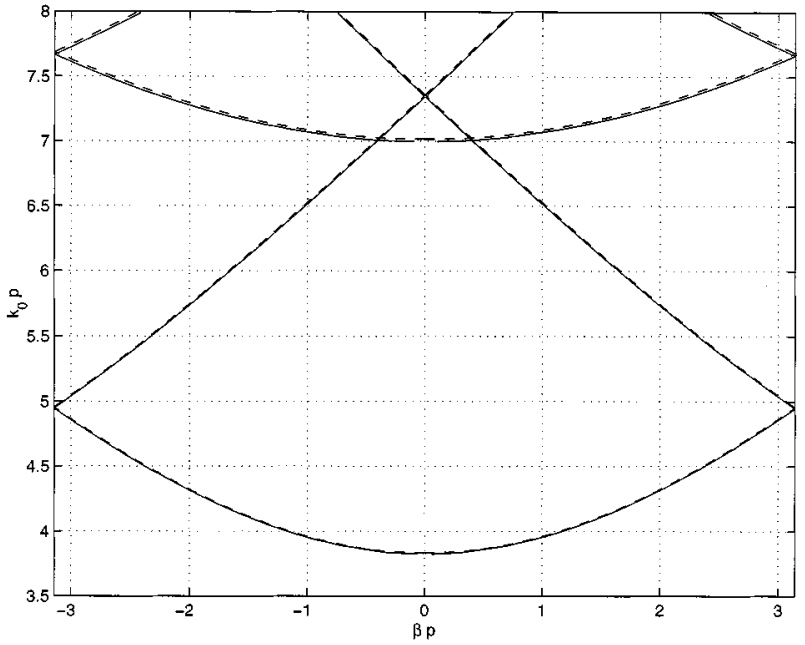

Fig. 7. $k_{0}-\beta$ diagram (solid line) of the structure in Fig. 1 when $\epsilon=1.01$, $b=0.5 a, p=0.5$, and $t=0.5 p$. Also shown is the analytical $k_{0}-\beta$ diagram (dashed line) of an empty circular waveguide of the same dimensions.

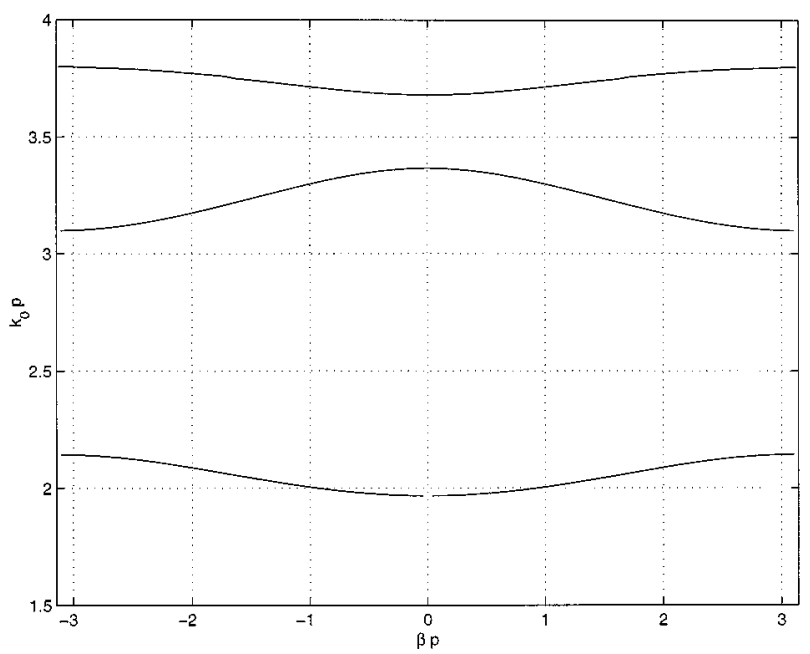

Fig. 8. $k_{0}-\beta$ diagram when $\epsilon=10, r_{1}=0.5 r_{0}, p=r_{0}$, and $t=0.5 p$.

\section{Capacitive Irises in Rectangular Waveguide}

As a third example, we consider a rectangular waveguide periodically loaded with capacitive irises [see Fig. 1(d)]. We focus on modes whose $x$-dependence is that of the fundamental mode of a rectangular waveguide, i.e., $\sin (\pi(x / a))$. For this structure, the normal modes of the two regions in the unit cell are taken as the longitudinal-section electric (LSE) modes instead of TE and TM modes [3].

Single irises and multiple irises in the same plane have been extensively investigated by Lewin using an integral-equation formulation [34]. Here, we are concerned with the interaction between multiple discontinuities of a periodic structure along the axis of the waveguide.

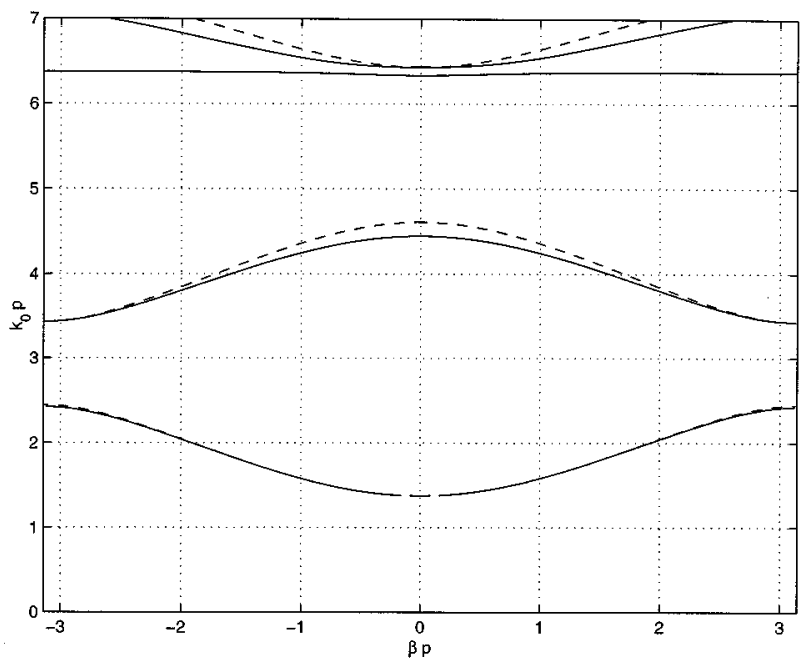

Fig. 9. $k_{0}-\beta$ of a rectangular waveguide loaded with capacitive irises. $t=$ 0 in, $a=0.9$ in, $b=0.2 \mathrm{in}, h=0.15 \mathrm{in}$, and $p=1 \mathrm{~cm}$. This study (solid line). From [3] (dashed line).

We examine the case of an infinitely thin iris to show that even this simple periodic structure can support complex modes. Fig. 9 shows the $k_{0}-\beta$ diagram when $t=0, b=0.2$ in, $a=0.9$ in, $p=1 \mathrm{~cm}$, and $h=0.15 \mathrm{in}$. The dashed lines are from the work of Collin $[3,387]$ and the solid lines are from this study. The agreement between the first two branches of the dispersion diagram is good, but noticeable deviations are present for higher branches. In fact, Collin states that: "For $k_{0}(p)>6.19$, the first higher-order mode begins to propagate, and consequently, the curves for $\beta_{c} l(p)$ have little significance for $k_{0}(p)$ greater than about $5, "[3$, p. 387]. It is indeed in this region that our results differ from his.

Fig. 10 shows the real and imaginary parts of the propagation constant as a function of frequency for the same dimensions as Fig. 9. The stars indicate complex modes. Complex modes in dielectric loaded waveguides have been extensively investigated by Omar and Schünemann [35], their existence in corrugated waveguides seems to have been first suggested by Cooper [36].

An intersting feature of the spectrum of this last structure is the existence of complex modes, with constant imaginary part of the propagation constant (see Fig. 10, dashed lines). In fact, these modes were also described by Collin for a capacitively loaded coaxial line where the eigenvalue equation for the propagation constant is easily shown to take the form

$$
\cosh (\gamma p)=\cos (\theta)-\frac{B}{2} \sin (\theta)
$$

Here, $\theta$ is the electric length of one period and $B$ is the normalized shunt susceptance ([37, p. 367]). When the right-hand side of this equation is negative and smaller that -1 , the possible solutions take the form [37, p. 367, eq. (8.10c)]

$$
\begin{aligned}
\operatorname{Re}[\gamma p] & =\cosh ^{-1}(-A) \\
\operatorname{Im}(\gamma p] & =\pi \\
A & =\cos (\theta)-\frac{B}{2} \sin (\theta)<-1 .
\end{aligned}
$$




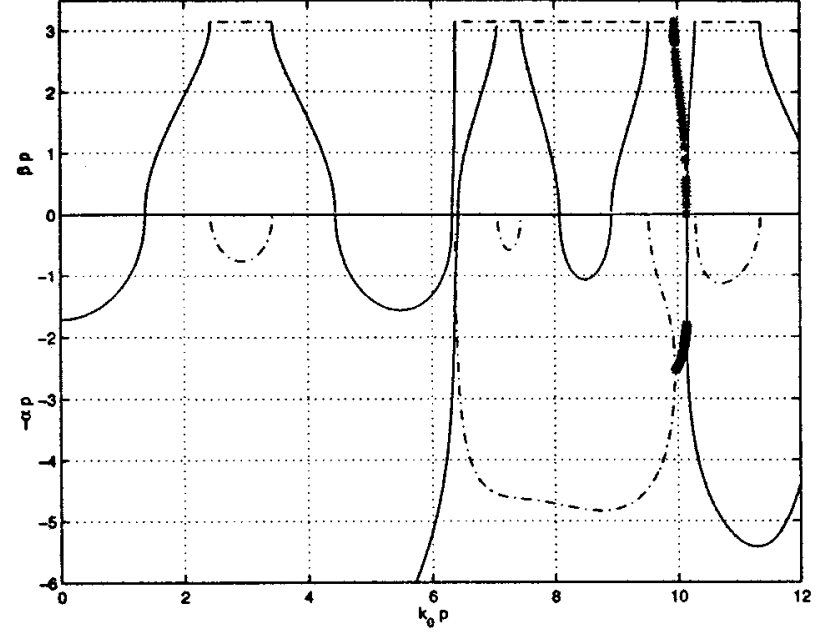

Fig. 10. $\gamma$ versus frequency for a rectangular waveguide loaded with capacitive irises. $t=0$ in, $a=0.9$ in, $b=0.2$ in, $h=0.15$ in, and $p=1 \mathrm{~cm}$. The stars show the complex modes in this structure.

In other words, the propagation constant is complex. The straight lines in the imaginary part of the propagation constant in Fig. 10 represent these solutions and provide an additional validation of the present formulation.

\section{CONCLUSION}

The propagation properties of corrugated and periodically loaded waveguides has been investigated using a formulation leading to a classical canonical matrix eigenvalue problem for the propagation constants. Complex, evanescent, and propagating modes were accurately determined for corrugated circular and rectangular waveguides, as well as a circular waveguide loaded with dielectric disks. Excellent agreement between the results of this study and previously published results was documented. The speed of the approach allows the determination of the entire dispersion diagram of corrugated waveguides with minimum numerical effort.

\section{REFERENCES}

[1] L. Brillouin, Wave Propagation in Periodic Structures. New York: Dover, 1953.

[2] D. A. Watkins, Topics in Electromagnetic Theory. New York: Wiley, 1958.

[3] R. E. Collin, Field Theory of Guided Waves. New York: McGraw-Hill, 1960.

[4] A. F. Harvey, Microwave Engineering. New York: Academic , 1963.

[5] J. B. Davies and B. J. Goldsmith, "An analysis of general mode propagation and the pulse-shortening phenomenon in electron linear accelerators," Philips Res. Rep., vol. 23, pp. 207-232, 1968.

[6] P. J. B. Clarricoats and M. I. Sobhy, "Propagation behavior of periodically loaded waveguides," Proc. Inst. Elect. Eng., vol. 115, pp. 652-661, 1968.

[7] G. H. Bryant, "Propagation in corrugated waveguides," Proc. Inst. Elect. Eng., vol. 116, pp. 203-213, 1969.

[8] P. J. B. Clarricoats and P. K. Saha, "Propagation and radiation behavior of corrugated feeds," Proc. Inst. Elect. Eng., vol. 118, pp. 1167-1176, Sept. 1971.

[9] A. M. B. Al-Hariri, A. D. Olver, and P. J. B. Clarricoats, "Low-attenuation properties of corrugated rectangular waveguides," Electron. Lett., vol. 10, no. 15, p. 304-3-5, July 1974.
[10] M. S. Navarro, T. E. Rozzi, and Y. T. Lo, "Propagation in a rectangular waveguide periodically loaded with resonant irises," IEEE Trans. Microwave Theory Tech., vol. MTT-28, pp. 857-865, Aug. 1980.

[11] G. L. James, "Analysis and design of $\mathrm{TE}_{11}$-to-HE $\mathrm{H}_{11}$ corrugated cylindrical waveguide mode converters using ring loaded slots," IEEE Trans. Microwave Theory Tech., vol. MTT-29, pp. 1059-1066, 1981.

[12] J. B. Davies, F. A. Fernandez, and G. Y. Philipou, "Finite-element analysis of all modes in cavities with circular symmetry," IEEE Trans. Microwave Theory Tech., vol. MTT-30, pp. 1975-1982, Nov. 1982.

[13] P. J. B. Clarricoats and A. D. Olver, Corrugated Horns for Microwave Antennas, Stevenage, U.K.: Peregrinus, 1984.

[14] A. D. Olver, K. K. Yang, and P. J. B. Clarricoats, "Propagation and radiation behavior of dual depth corrugated horns," Proc. Inst. Elec. Eng., pt. H, vol. 131, pp. 179-185, June 1984.

[15] J. Esteban and J. M. Rebollar, "Characterization of corrugated waveguides by modal analysis," IEEE Trans. Microwave Theory Tech., vol. 39, pp. 937-943, June 1991.

[16] A. D. Olver, P. J. B. Clarricoats, A. A. Kishk, and L. Shafai, Microwave Horns and Feeds. Piscataway, NJ: IEEE Press, 1994.

[17] Md. R. Amin, K. Ogura, H. Kitamura, K. Minami, T. Wannabe, Y. Carmel, W. Main, J. Weaver, W. W. Destler, and V. L. Granatstein, "Analysis of the electromagnetic waves in an overmoded finite length slow wave structure," IEEE Trans. Microwave Theory Tech., vol. 43, pp. 815-822, Apr. 1995.

[18] T. Itoh, G. Pelosi, and P. P. Silvester, Eds., Finite Element Software for Microwave Engineering. New York: Wiley, 1996.

[19] J. Modern Opt., vol. 41, Feb. 1994.

[20] C. M. Soukoulis, Ed., Photonic Band Gap Materials. Norwell, MA: Kluwer, 1996.

[21] J. D. Joannopoulos, R. D. Meade, and J. N. Winn, Photonic Crystals. Princeton, NJ: Princeton Univ. Press, 1995.

[22] V. Radisic, Y. Qian, and T. Itoh, "Broad-band power amplifier using dielectric photonic bandgap structure," IEEE Microwave Guided Wave Lett., vol. 8, pp. 13-14, Jan. 1998.

[23] V. Radisic, Y. Qian, R. Coccioli, and T. Itoh, "Novel 2-D photonic bandgap structure for microstrip lines," IEEE Microwave Guided Wave Lett., vol. 8, pp. 69-71, Feb. 1998.

[24] S. Amari, J. Bornemann, and R. Vahldieck, "Accurate analysis of scattering from multiple waveguide discontinuities using the coupled-integral-equation technique," J. Electromag. Waves Applicat., vol. 10, pp. 1623-1644, Dec. 1996.

[25] S. Amari, J. Bornemann, and R. Vahldieck, "Fast and accurate analysis of waveguide filters by the coupled-integral-equation technique," IEEE Trans. Microwave Theory Tech., vol. 45, pp. 1611-1618, Sept. 1997.

[26] S. Amari, R. Vahldieck, and J. Bornemann, "Analysis of propagation in periodically loaded circular waveguides," Proc. IEE Microwave Antennas Propagat., vol. 146, pp. 50-54, Feb. 1999.

[27] T. Itoh, Ed., Numerical Techniques for Microwave and Millimeter-Wave Passive Structures. New York: Wiley, 1989.

[28] R. F. Harrington, Time-Harmonic Electromagnetic Fields. New York: McGraw-Hill, 1961.

[29] S. Amari, R. Vahldieck, and J. Bornemann, "Accurate analysis of periodic structures with an additional symmetry in the unit cell from classical matrix eigenvalues," IEEE Trans. Microwave Theory Techn., vol. 46, pp. 1513-1515, Oct. 1998.

[30] R. F. Harrington, Field Computation by Moment Methods. Malabar, FL: Krieger, 1987.

[31] S. Amari, R. Vahldieck, J. Bornemann, and P. Leuchtmann, "Propagation in circular waveguide periodically loaded with dielectric disks," in IEEE MTT-S Int. Microwave Symp. Dig., Baltimore, MD, 1998, pp. $1143-1147$.

[32] T. Kitazawa, Y. Hayashi, and M. Suzuki, "Analysis of the dispersion characteristics of slot line with thick metal coating," IEEE Trans. Microwave Theory Tech., vol. MTT-28, pp. 387-392, Apr. 1980.

[33] S. Amari, A. Motamedi, J. Bornemann, and R. Vahldieck, "Global edge-conditioned basis functions from local solutions of Maxwell's equations," in IEEE MTT-S Int. Microwave Symp. Dig., June 1997, pp. 1373-1376.

[34] L. Lewin, Theory of Waveguides, London, U.K.: Newnes-Butterworths, 1975.

[35] A. S. Omar and K. F. Schüneman, "Complex and backward-wave modes in inhomogeneously and anisotropically filled waveguides," IEEE Trans. Microwave Theory Tech., vol. MTT-35, pp. 268-275, Mar. 1987.

[36] D. N. Cooper, "Complex propagation coefficients and the step discontinuity in corrugated cylindrical waveguide," Electron. Lett., vol. 7, pp. 135-136, Mar. 1971. 
[37] E. E. Collin, Foundations for Microwave Engineering. New York: McGraw-Hill, 1966.

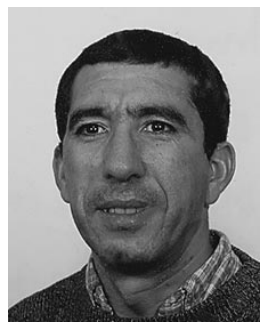

Smain Amari (M'98) received the D.E.S. degree in physics and electronics from Constantine University, Constantine, Algeria, in 1985, and the M.S. degree in electrical engineering and $\mathrm{Ph} . \mathrm{D}$. degree in physics from Washington University, St. Louis, MO, in 1989 and 1994, respectively.

He is interested in numerical methods in electromagnetics, numerical analysis, applied mathematics, applied physics, and application of quantum-field theory in quantum many-particle systems. Since 1994, he has been with the Department of Electrical and Computer Engineering, University of Victoria, Victoria, B.C., Canada.

Rüdiger Vahldieck (M'85-SM'86-F'99) received the Dipl.-Ing. and Dr.-Ing. degrees in electrical engineering from the University of Bremen, Bremen, Germany, in 1980 and 1983, respectively.

From 1984 to 1986, he was a Research Associate at the University of Ottawa, Ottawa, Ont., Canada. In 1986, he joined the Department of Electrical and Computer Engineering, University of Victoria, B.C., Canada, where he became a Full Professor in 1991. During Fall and Spring 1992-1993, he was a Visiting Scientist at the Ferdinand-Braun-Institute für Hochfrequenztechnik, Berlin, Germany. Since 1997, he has been a Professor of field theory at the Swiss Federal Institute of Technology, Zürich, Switzerland. He has published over 170 technical papers in books, journals, and conferences, mainly in the field of microwave computer-aided design (CAD). His research interests include numerical methods to model electromagnetic fields in the general area of electromagnetic compatibility (EMC) and, in particular, for computer-aided design of microwave, millimeter wave, and opto-electronic integrated circuits.

Prof. Vahldieck is the president of the IEEE 2000 International Zürich, Seminar on Broadband Communications (IZS'2000) and vice president of the EMC Congress, Zürich, Switzerland. He is a member of the Editorial Board of the IEEE TRANSACtion ON MicrowaVE THEORY AND TEChNiQues. Since 1992, he has also served on the Technical Program Committee of the IEEE International Microwave Symposium, the IEEE Microwave Theory and Techniques Society (IEEE MTT-S) Technical Committee on Microwave Field Theory and, in 1999, on the Technical Program Committee of the European Microwave Conference. $\mathrm{He}$ is the chairman of the IEEE Swiss Joint Chapter on IEEE MTT-S, IEEE AP-S, and IEEE EMC-S. Together with three co-authors, he received the 1983 Outstanding Publication Award presented by the Institution of Electronic and Radio Engineers. In 1996, he received the 1995 J. K. Mitra Award of the Institution of Electronics and Telecommunication Engineers (IETE) for the Best Research Paper.

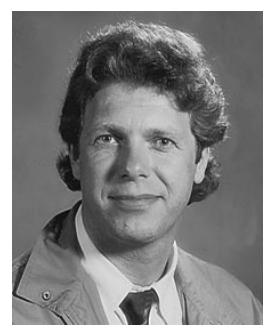

Jens Bornemann (M'87-SM'90) received the Dipl.-Ing. and Dr.-Ing. degrees from the University of Bremen, Bremen, Germany, in 1980 and 1984, respectively, both in electrical engineering.

From 1984 to 1985, he was a Consulting Engineer. In 1985, he joined the University of Bremen, as an Assistant Professor. Since April 1988, he has been with the Department of Electrical and Computer Engineering, University of Victoria, Victoria, B.C., Canada, where he became a Professor in 1992. From 1992 to 1995, he was a Fellow of the British Columbia Advanced Systems Institute. In 1996, he was a Visiting Scientist at Spar Aerospace Limited, Ste-Anne-de-Bellevue, Quebec, P.Q., Canada, and a Visiting Professor at the Microwave Department, University of Ulm, Ulm, Germany. Since 1997, he has been a Co-Director of the Center for Advanced Materials and Related Technology (CAMTEC), University of Victoria. He has co-authored Waveguide Components for Antenna Feed Systems. Theory and CAD (Norwood, MA: Artech House, 1993) and has authored/co-authored over 150 technical papers. His research activities include microwave/millimeter-wave components and systems design, and problems involving electromagnetic-field theory in integrated circuits, waveguide feed networks, and radiating structures. He serves on the Editorial Advisory Board of the International Journal of Numerical Modeling.

Dr. Bornemann is a Registered Professional Engineer in the Province of British Columbia, Canada. He is a senior member of IEEE Microwave Theory and Techniques and IEEE Antennas and Propagation Societies. He currently serves as an associate editor for the IEEE TRANSACTION ON MICROWAVE THEORY AND TECHNIQUES in the area of microwave modeling and CAD. He was a recipient of the 1983 A. F. Bulgin Premium presented by the Institution of Electronic and Radio Engineers.

Pascal Leuchtmann was born in Switzerland, in 1956. He received the diploma in electrical engineering and the Ph.D. degree from the Swiss Federal Institute of Technology (ETH), Zürich, Switzerland, in 1980 and 1987, respectively.

He then became a Scientific Assistant in the Electromagnetics Group, where he was involved with theoretical electrotechnics, mainly general-purpose numerical calculation of electromagnetic fields. From 1982 to 1988, he was a member of the CCITT Study Group V/GRD/6 (EMC powerlines versus telecom systems). He is currently a Lecturer of electromagnetics at $\mathrm{ETH}$, where he is engaged in EMC, microwaves, and antenna topics, mainly from the computational point-of-view. 